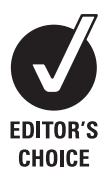

Department of Cardiovascular Medicine, Institute of Postgraduate Medical, Education and Research, Northwick Park Hospital, Harrow, UK

\section{Correspondence to:}

Professor R Senior, Department of Cardiovascular Medicine, Northwick Park Hospital, Harrow, Middlesex HA1 3UJ, UK; roxysenior@ cardiac-research.org

Accepted 27 November 2008 Published Online First 8 April 2009

\title{
Independent value of left atrial volume index for the prediction of mortality in patients with suspected heart failure referred from the community
}

\author{
T K Lim, G Dwivedi, S Hayat, S Majumdar, R Senior
}

\section{ABSTRACT}

Background: The left atrial volume index (LAVI) reflects left ventricular (LV) filling pressure and has been shown to predict outcome in various cardiovascular diseases. However, its value for the prediction of mortality in patients referred for suspected heart failure (HF) is unknown.

Objective: To assess the value of LAVI for the prediction of mortality independently of clinical, electrocardiographic (ECG) and echocardiographic prognostic parameters in patients with suspected HF referred from the community. Methods: 356 (mean (SD) age 72 (13) years) patients with suspected HF referred from the community were followed up for mortality after undergoing clinical assessment, ECG and echocardiography, including Doppler, to assess LV filling.

Results: Data were obtained for 335/356 (94\%) patients (162 male, 173 female) over a mean (SD) follow-up period of 30 (10) months, during which $38(11.3 \%)$ died. The univariate predictors for all-cause mortality were age, symptom of leg swelling, clinical signs of HF, abnormal ECG, LV ejection fraction, LAVI, LV end-systolic (LVESD) and diastolic dimension, septal wall thickness and the presence of other significant cardiac abnormalities. The only independent predictors of mortality were age (hazard ratio $(H R)=2.15,95 \% \mathrm{Cl} 1.42$ to $3.25, p<0.001)$, symptom of leg swelling $(\mathrm{HR}=2.83,95 \% \mathrm{Cl} 1.43$ to 5.59 , $\mathrm{p}=0.005)$, LAVI (HR $=1.25,95 \% \mathrm{Cl} 1.01$ to 1.54 , $\mathrm{p}=0.04)$ and LVESD (HR=1.32,95\% Cl 1.02 to 1.70 , $p=0.04)$.

Conclusion: LAVI provided independent information over clinical and other echocardiographic variables for predicting mortality in patients with suspected HF referred from the community.

Heart failure (HF) is a common disabling disease with high mortality and morbidity. It has been observed that among patients referred for suspected HF from the community almost $50 \%$ of patients with HF demonstrated normal left ventricular ejection (LVEF). ${ }^{12}$ Thus, measurement of LVEF alone is likely to underdiagnose $\mathrm{HF}$. Mortality, morbidity and hospitalisation in patients with HF and normal LVEF are similar to those found with reduced LVEF. ${ }^{34}$ In these patients, many authors have recognised the presence of significant abnormalities of diastolic function. ${ }^{5}$

Left atrial (LA) size, particularly the LA volume, has been recognised as a marker of diastolic dysfunction. ${ }^{6-10}$ Contrary to Doppler parameters, LA volume is independent of acute volume load, and therefore can provide a more accurate assessment of the duration and severity of left ventricular (LV) diastolic function. ${ }^{8}$ Recently, we and others have shown that LA volume is a powerful predictor of outcome in patients with ischaemic cardiomyopathy. ${ }^{11} 12$ Furthermore, we have also previously demonstrated that LA volume indexed (LAVI) to body surface area is a reliable indicator of LV filling pressure in patients presenting with features suggestive of HF but with preserved LVEF. ${ }^{13}$

In the United Kingdom, the major source of referral for echocardiography for suspected HF is from the community. There is a paucity of data on the ability of LAVI to predict mortality (robust marker of outcome in HF) in such patients. Therefore, the aim of this study was to evaluate the significance of LAVI in predicting all-cause mortality in patients with suspected HF referred from the community.

\section{PATIENTS AND METHODS \\ Patient selection}

Patients who were referred to our community echocardiography service by general practitioners for suspected HF (at least one symptom and/or one sign of $\mathrm{HF}$ as stated below) underwent clinical assessment, electrocardiography (ECG) and echocardiography. The symptoms considered were shortness of breath, fatigue or ankle swelling. Clinical signs of HF considered were raised jugular venous pressure, peripheral oedema, hepatomegaly, basal inspiratory crepitation or gallop rhythm. Patients with metastatic cancer or inability to cooperate as a result of mental incapacity such as dementia were excluded. All patients in the study were followed up for mortality. The research ethics committee approved the study.

\section{ECG}

An ECG was performed in the general practitioner surgery but was read by hospital doctor. An abnormal ECG was defined as the presence of atrial fibrillation (AF) or flutter, ventricular arrhythmia (ventricular tachycardia or multiple ventricular etopics) intraventricular conduction defects, ST- or T-wave abnormalities, pathological Q wave, paced rhythm or LV hypertrophy (voltage criteria).

\section{Echocardiography}

Echocardiographic images were acquired in the standard parasternal and apical views by experienced sonographers using the Cypress (Acuson, Mountain View, California, USA) ultrasound system. LVEF was assessed by a two-dimensional 
Table 1 Clinical characteristics and echocardiographic parameters of patients with and without event (mortality)

\begin{tabular}{|c|c|c|c|c|}
\hline Variables & $\begin{array}{l}\text { Total } \\
(\mathrm{n}=335)\end{array}$ & $\begin{array}{l}\text { Survivors } \\
(\mathrm{n}=297)\end{array}$ & $\begin{array}{l}\text { Non-survivors } \\
(\mathrm{n}=\mathbf{3 8})\end{array}$ & p Value* \\
\hline \multicolumn{5}{|l|}{ Clinical characteristics } \\
\hline Age (years) & $73(13)$ & $72(12)$ & $83(9)$ & $<0.001$ \\
\hline Male, n (\%) & $162(48)$ & $139(47)$ & $23(61)$ & 0.16 \\
\hline Body mass index $\left(\mathrm{kg} / \mathrm{m}^{2}\right)$ & $29(7)$ & $29(7)$ & $26(6)$ & 0.29 \\
\hline Systolic BP (mm Hg) & $141(26)$ & $141(21)$ & $142(24)$ & 0.8 \\
\hline Diastolic BP (mm Hg) & $79(14)$ & $79(13)$ & $81(22)$ & 0.30 \\
\hline Abnormal ECG, n (\%) & $194(58)$ & $164(55)$ & $30(79)$ & 0.009 \\
\hline \multicolumn{5}{|l|}{ Past medical history } \\
\hline Diabetes, n (\%) & $48(14)$ & 43 (14) & $5(13)$ & 0.98 \\
\hline Hypertension, n (\%) & $187(56)$ & $165(56)$ & $22(58)$ & 0.92 \\
\hline Ischaemic heart disease, $\mathrm{n}(\%)$ & $75(22)$ & $66(22)$ & $9(24)$ & 0.10 \\
\hline \multicolumn{5}{|l|}{ Symptoms and signs of HF } \\
\hline Shortness of breath, n (\%) & $271(81)$ & $239(80)$ & $32(84)$ & 0.73 \\
\hline Fatigue, n (\%) & $147(44)$ & $126(42)$ & $21(55)$ & 0.18 \\
\hline Swelling of legs, n (\%) & $130(39)$ & $106(36)$ & $24(63)$ & 0.002 \\
\hline Clinical signs of HF, $\mathrm{n}(\%)$ & $172(51)$ & $145(49)$ & $27(71)$ & 0.02 \\
\hline \multicolumn{5}{|l|}{ Medication } \\
\hline Diuretic, n (\%) & $154(46)$ & $130(44)$ & $24(63)$ & 0.04 \\
\hline ACEI or angiotensin blocker, $\mathrm{n}(\%)$ & $115(34)$ & $100(34)$ & $15(39)$ & 0.47 \\
\hline$\beta$ Blocker, $\mathrm{n}(\%)$ & $64(19)$ & $61(21)$ & $3(8)$ & 0.10 \\
\hline Aspirin/clopidogrel, n (\%) & $121(36)$ & $107(36)$ & $14(37)$ & 0.93 \\
\hline \multicolumn{5}{|l|}{ Echocardiographic parameters } \\
\hline $\operatorname{LVEF}(\%)$ & $58(13)$ & $58(12)$ & $52(15)$ & 0.002 \\
\hline $\mathrm{E} / \mathrm{A}$ & $0.97(0.50)$ & $0.98(0.48)$ & $0.96(0.67)$ & 0.85 \\
\hline LVEDD (cm) & $4.9(0.9)$ & $4.9(0.8)$ & $5.3(1.2)$ & 0.004 \\
\hline LVESD (cm) & $3.1(1.0)$ & $3.0(0.9)$ & $3.7(1.4)$ & $<0.001$ \\
\hline PW thickness $(\mathrm{cm})$ & $1.0(0.2)$ & $1.0(0.2)$ & $1.1(0.2)$ & 0.12 \\
\hline IVS thickness (cm) & $1.1(0.3)$ & $1.1(0.3)$ & $1.3(0.3)$ & 0.02 \\
\hline LAVI $\left(\mathrm{ml} / \mathrm{m}^{2}\right)$ & $23(13)$ & $22(11)$ & $34(20)$ & $<0.001$ \\
\hline Deceleration time (ms) & $177(67)$ & $179(67)$ & $163(66)$ & 0.40 \\
\hline
\end{tabular}

Results are shown as mean (SD) unless stated otherwise.

${ }^{*}$ Comparing the variables between survivors and non-survivors.

ACEl, ACE inhibitor; BP, blood pressure; HF, heart failure; IVS, interventricular septal; LAVI, left atrial volume index; LVEDD, left ventricular end-diastolic dimension; LVEF, left ventricular ejection fraction; LVESD, left ventricular end-systolic dimension; PW, posterior wall.

visual estimation method which had been previously validated against quantitative LVEF both by our group and others. ${ }^{5}{ }^{14} 15$ LA volume was calculated using the formula for an ellipsoid ${ }^{16}$ :

$$
\text { LA volume }(m l)=\frac{\pi}{6}\left\{D_{1} D_{2} D_{3}\right\}
$$

where $\mathrm{D}$ is the LA dimension obtained at end systole from a parasternal long-axis $\left(D_{1}\right)$ and apical four-chamber view (horizontal $\left(\mathrm{D}_{2}\right)$ and anteroposterior measurements $\left(\mathrm{D}_{3}\right)$ ). LAVI was calculated as LA volume/body surface area. LV diastole filling pattern was assessed by placing the pulse Doppler sample volume at the tips of the mitral valve leaflets. From the transmitral recording, peak E velocity (peak early transmitral filling velocity during early diastole) and peak A velocity (peak transmitral atrial filling velocity during late diastole) were measured in centimetres per second. Thereafter, the ratio of $\mathrm{E}$ and $\mathrm{A}$ wave was calculated. Deceleration time of the $\mathrm{E}$ wave was also calculated as the time (in milliseconds) between peak E velocity and the point where the extrapolation of the deceleration slope of E velocity crosses the zero baseline.
LV wall thickness was measured as interventricular septal and posterior wall thickness at end diastole, whereas LV dimensions were determined at end systole (LVESD) and diastole (LVEDD) in the parasternal long- or short-axis view. Echocardiographic evidence of LV hypertrophy was defined as either interventricular septal or posterior wall thickness $\geqslant 1.3 \mathrm{~cm} .{ }^{17}$ Significant valvular heart disease was defined as any evidence of at least moderate aortic stenosis, aortic regurgitation, mitral stenosis and mitral regurgitation (mitral valve disease) based on colour and Doppler echocardiography. Other significant cardiac abnormalities were defined as the presence of significant valvular heart disease or isolated right ventricular dysfunction or isolated raised pulmonary artery pressure $\geqslant 60 \mathrm{~mm} \mathrm{Hg}$.

\section{Outcome}

Patients were followed up for all-cause mortality. Follow-up data were collected through the Hospital Integrated Customer Services System (Silverlink Software, Newcastle upon Tyne, United Kingdom) and by questionnaires returned from patients, with additional telephone calls or hospital record review to further verify events where appropriate. Follow-up time was 
Table 2 Univariable predictors for all-cause mortality

\begin{tabular}{l} 
Variables \\
\hline Clinical characteristics \\
Age $^{*}$ (years) \\
Sex (male) \\
Systolic blood pressure* $(\mathrm{mm}$ \\
Diastolic blood pressure* $(\mathrm{mm}$ \\
Body mass index* $\left(\mathrm{kg} / \mathrm{m}^{2}\right)$ \\
Symptoms and signs of HF \\
Shortness of breath \\
Fatigue \\
Swelling of legs \\
Clinical signs of $\mathrm{HF}$
\end{tabular}

Past medical history

Ischaemic heart disease

Diabetes mellitus

Hypertension

Abnormal ECG

\section{Hazard ratio}

$(95 \% \mathrm{Cl})$

p Value

$2.83(1.92$ to 4.20$)$

1.68 (0.88 to 3.23$)$

1.02 (0.87 to 1.12$)$

1.17 (0.90 to 1.53$)$

0.75 (0.44 to 1.29 )

$<0.001$

0.12

0.82

0.24

0.31

$1.32(0.55$ to 3.15$)$

$1.61(0.85$ to 3.06$)$

2.91 (1.51 to 5.63$)$

2.44 (1.21 to 4.93 )

1.08 (0.51 to 2.29$)$

0.95 (0.37 to 2.43 )

1.12 (0.59 to 2.14$)$

2.85 (1.31 to 6.22 )

0.53

0.14

0.001

0.01

Echocardiographic parameters

$\operatorname{LVEF}^{*}(\%)$

$\operatorname{LAVI}^{*}\left(\mathrm{ml} / \mathrm{m}^{2}\right)$

Deceleration time $\uparrow(\mathrm{ms})$

E/A:

Septal wall thickness§ $(\mathrm{cm})$

Posterior wall thickness§ $(\mathrm{cm})$

LV dimension in diastole§ $(\mathrm{cm})$

LV dimension in systole§ $(\mathrm{cm})$

Other significant echocardiographic

abnormalities

*Hazard ratios given for a 10-unit increase in explanatory variable; †hazard ratios given for a 100-unit increase in explanatory variable; tvariable analysed on the log scale; §hazard ratios given for a 1-unit increase in explanatory variable.

$H F$, heart failure; LAVI, left atrial volume index; LVEF, left ventricular ejection fraction.

calculated from the time of echocardiography to either the event date or to the date of last contact with the patients.

\section{Statistical methods}

Results from normally distributed continuous data are shown as mean (SD) and categorical data as percentages (\%). Categorical data were analysed by the $\chi^{2}$ test and normally distributed continuous data by the Student $t$ test. The best cut-off points for LAVI and LVESD to predict mortality were derived from the receiver operating characteristic curve. The effects of known prognostic clinical, ECG and echocardiographic variables upon the outcome were examined using Cox proportional hazards regression analysis. Analysis of the data was performed in two stages. Initially, the individual effects of clinical, ECG and echocardiographic variables were examined separately in a series of univariate analyses. Subsequently, the joint effect of the explanatory variables upon the time to mortality was examined in a multivariable analysis. A backwards selection procedure was used to retain only the statistically significant variables. This method involved removing non-significant variables from the analysis, one at a time, until all remaining variables were statistically significant. In addition, Kaplan-Meier curves were generated and the differences between survival distributions were assessed using the log-rank test. A p value of $<0.05$ (twosided) was considered significant. Statistical analysis was performed with Analyse-it software for Microsoft excel (version
Table 3 Independent predictors for all-cause mortality

\begin{tabular}{llc}
\hline Variables & $\begin{array}{l}\text { Hazard ratio } \\
\text { (95\% } \mathbf{~ C l )}\end{array}$ & p Value \\
\hline Age $^{*}$ (years) & $2.15(1.42$ to 3.25$)$ & $<0.001$ \\
Leg swelling & $2.83(1.43$ to 5.59$)$ & 0.005 \\
LAVI $^{*}\left(\mathrm{ml} / \mathrm{m}^{2}\right)$ & $1.25(1.01$ to 1.54$)$ & 0.04 \\
LVESD $^{\prime}(\mathrm{cm})$ & $1.32(1.02$ to 1.70$)$ & 0.04 \\
\hline
\end{tabular}

*Hazard ratios given for a 10-unit increase in explanatory variable; thazard ratios given for a 1-unit increase in explanatory variable. LAVI, left atrial volume index; LVESD, left ventricular end-systolic dimension.

1.62, Analyse-it Software, Leeds, UK) and Stata statistical software (version 7, StataCorp, USA).

\section{RESULTS}

Patients

A total of 356 patients with suspected HF, referred by their general practitioner (February 2002 to April 2004) from the community, were evaluated. All patients underwent echocardiography within 14 days of referral. Table 1 shows the baseline characteristics and echocardiographic parameters of the study group. LV systolic dysfunction (LVEF $<50 \%$ ) was present in 64/ $335(19 \%)$ patients. Moderate-severe mitral valvular disease was present in only $10(3 \%)$ of patients and atrial flutter/fibrillation was found in $43(13 \%)$ of patients. Follow-up data were obtained in 335/356 (94\%) patients over a mean (SD) follow-up time of 30 (10) months. A total of $38(11.3 \%)$ patients died. Patients who died were older, more likely to have symptoms of leg swelling and signs of HF, more likely to be receiving a diuretic agent, with higher incidence of abnormal ECG, lower LVEF, larger LVESD, larger LAVI and higher incidence of LV hypertrophy than those who survived. There were no differences in other drug treatment in patients who died compared with those who survived.

\section{Univariable predictors of mortality (table 2)}

The univariate predictors for all-cause mortality were age, symptom of leg swelling, clinical signs of HF, abnormal ECG, LVEF, LAVI, LV dimensions, septal wall thickness and the presence of other significant cardiac abnormalities. Increased age was associated with an increased likelihood of mortality at any time. A 10-year increase in age resulted in the hazard of mortality at any time increasing by around 2.8 times. The presence of leg swelling, signs of HF and abnormal ECG were all associated with increased hazard of mortality at any time with a hazard ratio of 2.91, 2.44 and 2.85, respectively. Higher values of LAVI, septal wall thickness, LVESD and LVEDD were associated with a greater hazard of mortality at any time. For example, a 10-unit increase in LAVI increased the hazard of death by $50 \%$, while a 1 -unit increase in LV dimension increased the hazard of death by over $60 \%$. Conversely, a 10-unit reduction in LVEF was associated with an increased hazard of death by $30 \%$. Similarly, patients with other significant echocardiographic abnormalities were nearly four times more likely to die than patients without the presence of other significant echocardiographic abnormalities.

\section{Multivariable predictors of mortality (table 3)}

On multivariable Cox regression analysis, the independent predictors of mortality were age, symptom of leg swelling, LAVI and LVESD. Clinical signs of HF, abnormal ECG, and other echocardiographic parameters such as LVEF, septal wall thickness 
Table 4 Predictive values for mortality using echocardiography

\begin{tabular}{|c|c|c|c|c|c|}
\hline Predictive values & $\begin{array}{l}\text { LVEF } \\
<\mathbf{5 0} \%\end{array}$ & $\begin{array}{l}\text { LAVI } \\
>20 \mathrm{ml} / \mathrm{m}^{2}\end{array}$ & $\begin{array}{l}\text { LVESD } \\
>3 \mathrm{~cm}\end{array}$ & $\begin{array}{l}\text { LAVI } \\
>20 \mathrm{ml} / \mathrm{m}^{2} \\
\text { or LVESD } \\
>3 \mathrm{~cm}\end{array}$ & $\begin{array}{l}\text { Abnormal } \\
\text { echocardiography }\end{array}$ \\
\hline Sensitivity (\%) & 40 & 79 & 68 & 90 & 92 \\
\hline Specificity (\%) & 84 & 54 & 62 & 40 & 38 \\
\hline Positive predictive values (\%) & 23 & 18 & 19 & 16 & 16 \\
\hline Negative predictive values (\%) & 92 & 95 & 94 & 97 & 97.4 \\
\hline
\end{tabular}

LAVI, left atrial volume index; LVEF, left ventricular ejection fraction; LVESD, left ventricular end-systolic dimension.

and the presence of other significant cardiac abnormalities were not found to have a significant effect upon mortality. A 10-year increase in age resulted in the hazard of death increase by over twofold, whilst the presence of leg swelling increased the hazard of death by almost threefold. Similarly, a 10-unit increase in LAVI was associated with increased hazard of death by $25 \%$, while a 1 unit increase in LVESD resulted in an increased likelihood of death by $32 \%$. After adjusting for patients with AF and mitral valve disease (confounding factors for LA dilatation), LAVI remained an independent predictor of mortality $(p=0.03)$, in addition to age $(p<0.001)$, leg swelling $(p=0.03)$, LVESD $(p=0.02)$ and septal thickness $(p=0.03)$.

\section{Prediction of mortality by echocardiography (table 4)}

The sensitivity and specificity of LVEF $<50 \%$ for the prediction of mortality were $40 \%$ and $84 \%$, respectively. Using receiver operating characteristic analysis, the best cut-off points for LAVI and LVESD to predict mortality were found to be $20 \mathrm{ml} / \mathrm{m}^{2}$ (area under the curve $=0.72, p<0.001$ ) and $3 \mathrm{~cm}$ (area under the curve $=0.67, \mathrm{p}<0.001$ ), respectively, with significantly higher sensitivity but lower specificity than for patients with LVEF $<50 \%$. Figure 1 demonstrates Kaplan-Meier survival curves for patients with LAVI $>20 \mathrm{ml} / \mathrm{m}^{2}$ versus those patients with LAVI $\leqslant 20 \mathrm{ml} / \mathrm{m}^{2}$. A total of 119 out of 335 patients $(36 \%)$ demonstrated LAVI $\leqslant 20 \mathrm{ml} / \mathrm{m}^{2}$ and LVESD of $\leqslant 3.0 \mathrm{~cm}$. The presence of systolic (LVESD $>3 \mathrm{~cm}$ ) dysfunction or diastolic dysfunction (LAVI $>20 \mathrm{ml} / \mathrm{m}^{2}$ ) resulted in a sensitivity of $90 \%$ and a specificity of $40 \%$ for the prediction of mortality. A normal echocardiogram (LVEF $\geqslant 50 \%$, LAVI $\leqslant 20 \mathrm{ml} / \mathrm{m}^{2}$, LVESD $\leqslant 3 \mathrm{~cm}$ and without the presence of significant valvular heart disease or isolated right ventricular dysfunction

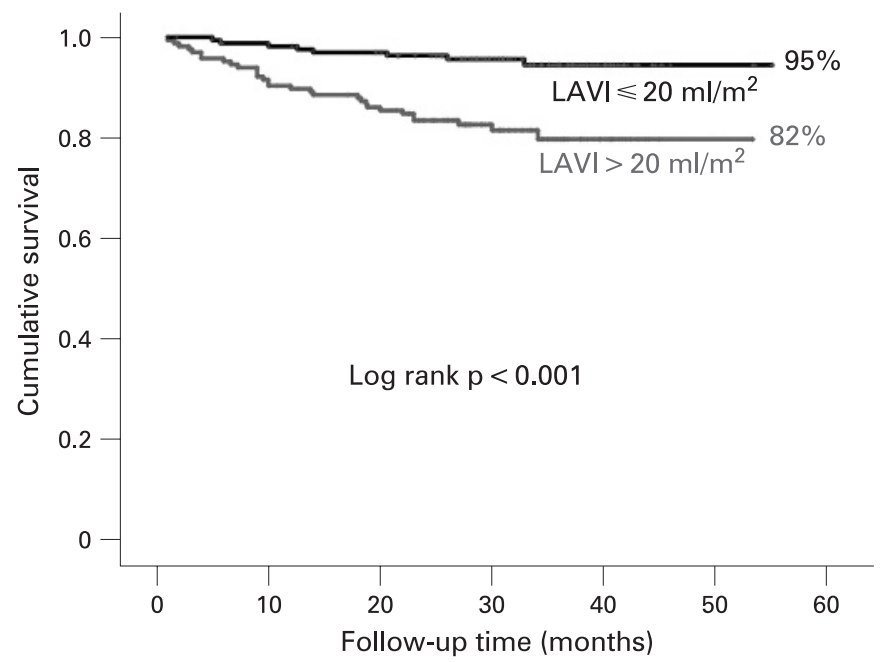

Figure 1 Kaplan-Meier survival curves in patients demonstrating a normal versus an increased left atrial volume index (LAVI). or isolated elevated pulmonary artery pressure $\geqslant 60 \mathrm{~mm} \mathrm{Hg}$ ) was present in $117(35 \%)$ and conferred an excellent prognosis with mortality of $2.6 \%$ compared with $16 \%$ in those without normal echocardiography $(p<0.001)$. Figure 2 demonstrates Kaplan-Meier survival curves for patients with and without normal echocardiography.

\section{Value of LAVI for the prediction of mortality in relation to drug treatment of heart failure}

LAVI remained a predictor of mortality in patients with $(p<0.05)$ and without $(p<0.001) \beta$ blocker therapy. While LAVI was a significant predictor of mortality in patients with HF not receiving treatment, no meaningful statistical data could be obtained in those receiving optimal medical treatment because only one patient died in this group.

Mortality in different categories of patients with suspected HF Among those who demonstrated no objective evidence of LV dysfunction (LVEF $\geqslant 50 \%$ and LAVI $\leqslant 20 \mathrm{ml} / \mathrm{m}^{2}$ ) mortality was only 7 out of $158(4 \%)$. This increased significantly $(\mathrm{p}<0.001)$ to $23 \%(15 / 64)$ in systolic HF (LVEF $<50 \%)$. Mortality also increased significantly $(p=0.004)$ to $14 \%(16 / 113)$ in patients with HF but normal LVEF (LAVI $>20 \mathrm{ml} / \mathrm{m}^{2}$ ). However, in the group of patients with systolic HF who did not demonstrate raised LV filling pressure (LAVI $\leqslant 20 \mathrm{ml} / \mathrm{m}^{2}$ ), mortality was reduced to $10 \%(1 / 10)$, but increased significantly $(p<0.001)$ to

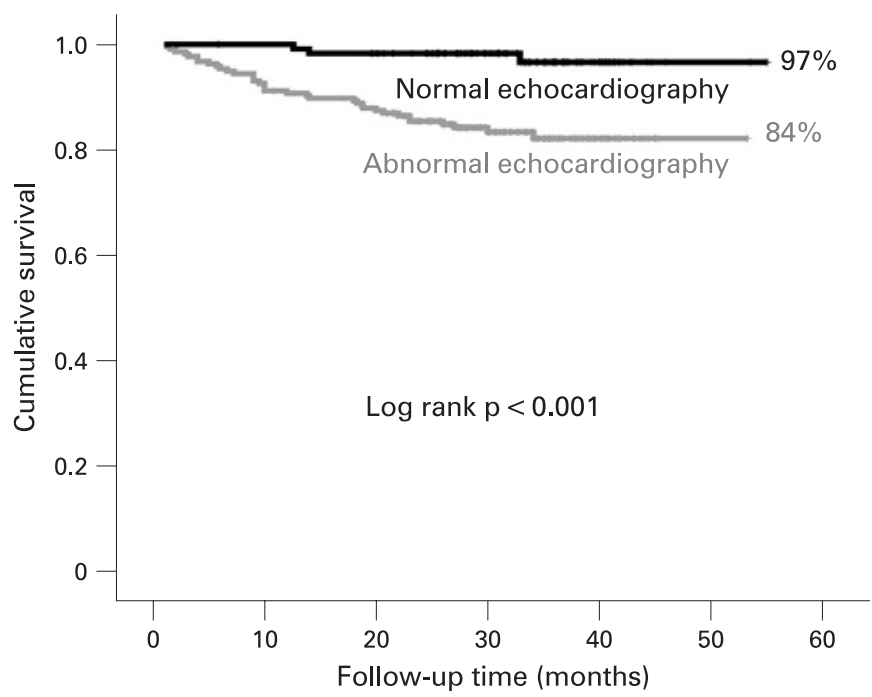

Figure 2 Kaplan-Meier survival curves in patients with and without normal echocardiography. A normal echocardiography is defined as left ventricular ejection fraction $\geqslant 50 \%$, left atrial volume index $\leqslant 20 \mathrm{ml} / \mathrm{m}^{2}$, left ventricular end-systolic dimension $\leqslant 3 \mathrm{~cm}$ and without the presence of significant valvular heart disease or isolated right ventricular dysfunction or isolated raised pulmonary artery pressure $\geqslant 60 \mathrm{~mm} \mathrm{Hg}$. 


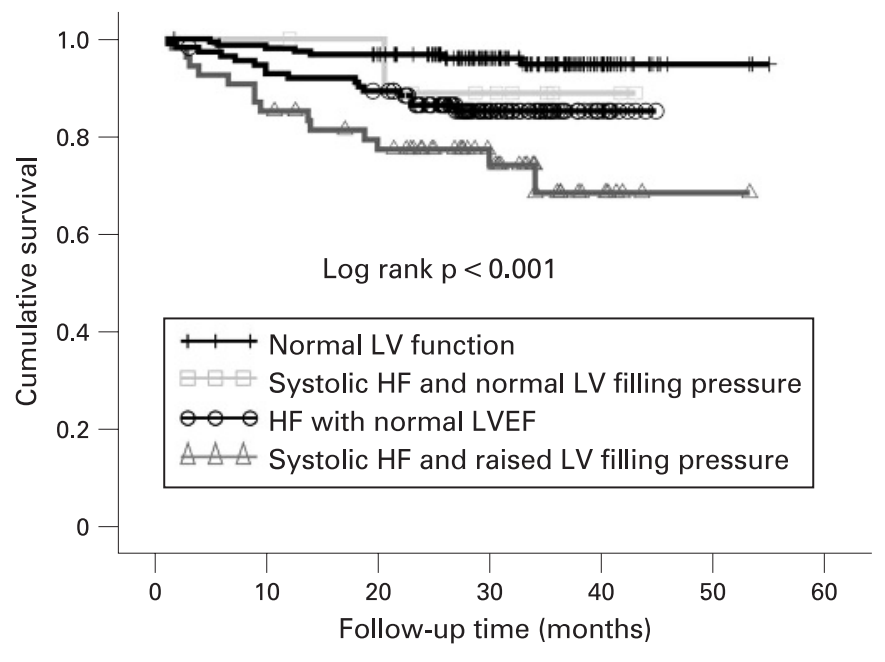

Figure 3 Kaplan-Meier survival curves for patients in various categories of heart failure (HF). LVEF, left ventricular ejection fraction.

$26 \%(14 / 54)$ when patients with systolic HF demonstrated raised LV filling pressure (LAVI $>20 \mathrm{ml} / \mathrm{m}^{2}$ ) compared with patients without LV dysfunction. Figure 3 demonstrates the Kaplan-Meier survival curves for the above groups. Table 5 illustrates the characteristics of patients with systolic HF versus HF with normal LVEF. HF with normal LVEF was more prevalent, had a greater preponderance of women, demonstrated higher systolic blood pressure, higher prevalence of hypertension but lower prevalence of ischaemic heart disease with smaller LV dimensions and greater LV wall thickness than patients with systolic HF.

\section{DISCUSSION}

As far as we know, this is the first study to assess the value of simple measurements of echocardiography for the prediction of mortality relative to known clinical and ECG prognostic markers in patients referred by the general practitioner for echocardiography for suspected HF. The study showed that LAVI, a marker of LV diastolic function, in particular raised LV filling pressure measured by echocardiography, independently predicted survival over and above clinical and ECG variables. This study also demonstrated that LVESD, a marker of LV systolic function, is also an independent measure of survival. In a population where mortality is about $11 \%$ over 2.5 years, an abnormal echocardiography which included LAVI and LVESD estimation predicted more than sixfold increase in mortality (16\%) compared with a normal echocardiography (2.6\%).

A completely normal echocardiogram was present in more than one-third of these patients with suspected HF and almost half of the patients demonstrated no objective evidence of cardiac dysfunction. The study also illustrated that in patients with suspected HF when there was no objective evidence of LV dysfunction mortality was only $4 \%$ but increased significantly almost fourfold and sixfold in patients with HF with normal LVEF and systolic HF, respectively. However, the mortality was highest $(26 \%)$ in patients with systolic HF demonstrating evidence of raised LV filling pressure but reduced dramatically to $10 \%$ when there was no evidence of raised LV filling pressure.

Thus, simple, quantitative echocardiographic measures that assessed both systolic and diastolic function can predict survival in patients referred by the general practitioner for suspected HF. LVEF, a marker of LV systolic function, though a strong
Table 5 Clinical characteristics and echocardiography parameters in patients with systolic heart failure (HF) versus HF with normal left ventricular ejection fraction (LVEF)

\begin{tabular}{lccc}
\hline & $\begin{array}{l}\text { Systolic HF } \\
\text { (n=64) }\end{array}$ & $\begin{array}{l}\text { HF with } \\
\text { normal LVEF } \\
\text { (n= 113) }\end{array}$ & p Value \\
\hline Clinical characteristics & & & \\
Age (years) & $78(10)$ & $77(9)$ & 0.4 \\
Male, n (\%) & $42(66)$ & $56(50)$ & 0.04 \\
Body mass index (kg/m²) & $27(5)$ & $28(5)$ & 0.12 \\
Systolic BP (mm Hg) & $136(21)$ & $143(22)$ & 0.03 \\
Diastolic BP (mm Hg) & $77(19)$ & $79(13)$ & 0.45 \\
Abnormal ECG, n (\%) & $59(92)$ & $70(79)$ & $<0.001$ \\
& & & \\
Past medical history & $11(17)$ & $17(15)$ & 0.70 \\
Diabetes, n (\%) & $29(45)$ & $70(62)$ & 0.03 \\
Hypertension, n (\%) & $24(38)$ & $25(22)$ & 0.03 \\
Ischaemic heart disease, $\mathrm{n}(\%)$ & &
\end{tabular}

Symptoms and signs of HF

Shortness of breath, $\mathrm{n}(\%)$

Fatigue, n (\%)

Swelling of legs, $\mathrm{n}(\%)$

$\begin{array}{lll}58(91) & 94(83) & 0.17 \\ 31(48) & 49(43) & 0.51 \\ 19(30) & 47(42) & 0.11 \\ 37(58) & 58(51) & 0.40\end{array}$

Clinical signs of $\mathrm{HF}, \mathrm{n}(\%)$

37 (58)

58 (51)

0.40

$\begin{array}{lccr}\text { Medication } & & & \\ \text { Diuretic, n (\%) } & 35(55) & 50(44) & 0.18 \\ \text { ACEl or angiotensin blocker, n (\%) } & 30(47) & 38(34) & 0.08 \\ \beta \text { Blocker, n (\%) } & 10(16) & 25(22) & 0.29 \\ \text { Aspirin/clopidogrel, n (\%) } & 27(42) & 38(34) & 0.25 \\ & & & \\ \text { Echocardiographic parameters } & & & \\ \text { LVEF (\%) } & 36(9) & 63(7) & <0.001 \\ \text { E/A } & 1.19(0.68) & 0.99(0.59) & 0.08 \\ \text { LVEDD (cm) } & 5.66(1.2) & 4.82(0.69) & <0.001 \\ \text { LVESD (cm) } & 4.47(1.23) & 2.91(0.59) & <0.001 \\ \text { PW thickness (cm) } & 1.0(0.2) & 1.1(0.2) & 0.12 \\ \text { IVS thickness (cm) } & 1.1(0.3) & 1.3(0.3) & 0.02 \\ \text { LAVI (ml/m²) } & 22(11) & 34(20) & <0.001 \\ \text { Deceleration time (ms) } & 179(67) & 163(66) & 0.40\end{array}$

Results are shown as mean (SD) unless stated otherwise. ACEI, ACE inhibitor; BP, blood pressure; IVS, interventricular septal; LAVI, left atrial volume index; LVEDD, left ventricular end-diastolic dimension; LVEF, left ventricular ejection fraction; LVESD, left ventricular end-systolic dimension; PW, posterior wall.

univariable predictor of mortality was not an independent predictor in our study. It has been previously shown that while a low LVEF $^{18}$ predicts mortality, a normal LVEF also results in significant mortality in patients with $\mathrm{HF} .{ }^{19}$ This is similar to our study where patients with normal LVEF had $8 \%$ mortality over 2.5 years in a population where the mortality rate is $11 \%$. However, when an additional marker of LV systolic function, like LVESD and diastolic function (LAVI), were assessed, the predictive value for outcome improved. That, addition of other markers of LV systolic function like LVESD provides incremental information about outcome in cardiac patients has been previously demonstrated in patients after acute myocardial infarction. ${ }^{20}$ White et al showed that assessment of LV endsystolic volume in each category of LVEF improved prediction of mortality. ${ }^{20}$ This is because physiological LV end-systolic parameters are more intimately related to wall stress than LVEF, which is also more load dependent.

A raised LAVI is a recognised marker of diastolic function, ${ }^{10}$ in particular a marker of raised LV filling pressure. Since the left atrium communicates with the left ventricle through an open 
mitral orifice in diastole, increased LV filling pressure raises wall tension and results in LA distension. While two-dimensional LA dimensions are technically easy to measure, LA volume has been proposed as a better index of LA enlargement since LA remodelling is associated with a reduced sphericity, and facilitates LA volume derivation from two-dimensional parameters. ${ }^{91}$ LA volume represents an integrated assessment of chronic LV filling pressure, negating the need to rely on point Doppler estimates that are subject to loading variation. Furthermore, Doppler estimates do not reliably predict LV filling pressure in patients with normal LVEF. ${ }^{22}$ In our population, the majority $(76 \%)$ had LVEF $>50 \%$ and none of the Doppler parameters predicted mortality. On the other hand, an extensive body of publications attest to the value of LAVI for both haemodynamic and prognostic assessment in a variety of settings. ${ }^{11}{ }^{23-25}$ However, to date there is a paucity of data about the value of LAVI for predicting mortality in patients referred for echocardiography in patients with suspected HF.

\section{Limitations}

LVEF was not assessed quantitatively in this study. This may have reduced the power of LVEF for the prediction of mortality. However, it has been shown in many studies that LVEF assessed qualitatively by experienced observers correlates closely with quantitative LVEF. ${ }^{514}$ Furthermore, in another study group similar to ours, quantitative LVEF did not predict major outcome. $^{26}$ The reason why LVEF did not emerge as an independent predictor of outcome in both studies is probably because most of the patients $(>75 \%)$ had a normal LVEF in these studies which may have reduced the power of the study to assess the predictive value of a low LVEF.

The study was also not powered to assess differences among HF subgroups. LV dimensions were not indexed to body surface area, while LA volume was. This was because according to American and British Society Echocardiography guidelines, LA volume should always be indexed but LV dimensions may not be indexed. ${ }^{17}{ }^{27}$ This is probably because there is a larger variation of LA volume with body surface area than for the LV dimension.

In this study, we did not assess serum B-type natriuretic peptide (BNP) levels, which are now measured routinely in patients with suspected HF. However, we did assess N-terminalproBNP in a subset of these patients (132 patients) and we demonstrated that N-terminal-proBNP and echocardiography, which incorporated LAVI, independently provided outcome information (combined mortality or readmission for $\mathrm{HF}$ ). ${ }^{28}$

Another potential limitation of the study is that the population consisted of patients with mitral valve disease and AF. These conditions may increase LAVI in the absence of increased LV filling pressure. However, in a population which is elderly these patients may well have associated increased LV filling pressure due to underlying myocardial disease. Indeed, these patients constitute a typical group referred by general practitioners for suspected HF. However, LAVI remained an independent predictor of mortality after adjusting for patients with $\mathrm{AF}$ and mitral valve disease.

Finally, we did not perform tissue Doppler imaging, which also determines LV filling pressure. Whether the tissue Doppler parameters provide incremental information over and above LAVI is yet to be determined.

\section{Clinical implication}

With advancement of ultrasound technology, it is now possible to perform point of care echocardiography with high accuracy compared with standard echocardiography. ${ }^{1429}$ 30 LAVI and LVESD can be easily measured. Our study demonstrated that incorporating LAVI and LVESD measurements improves prediction of outcome independent of clinical and other echocardiographic variables.

\section{CONCLUSION}

LAVI provided independent information over clinical variables for predicting mortality in patients with suspected HF referred from the community.

Acknowledgements: We are grateful to Mr Paul Basset who provided statistical support.

Funding: The work was supported by a grant from the Cardiac Research Fund, Northwick Park Hospital, Harrow, UK

Competing interests: None.

Ethics approval: Approval from Harrow Research Ethics Committee.

\section{REFERENCES}

1. Senni M, Tribouilloy CM, Rodeheffer RJ, et al. Congestive heart failure in the community: a study of all incident cases in Olmsted County, Minnesota, in 1991 Circulation 1998:98:2282-9.

2. Cowie MR, Wood DA, Coats AJ, et al. Incidence and aetiology of heart failure; a population-based study. Eur Heart J 1999:20:421-8.

3. McAlister FA, Teo KK, Taher M, et al. Insights into the contemporary epidemiology and outpatient management of congestive heart failure. Am Heart J 1999;138(Pt 1):87-94.

4. Bursi F, Weston SA, Redfield MM, et al. Systolic and diastolic heart failure in the community. JAMA 2006;296:2209-16.

5. Redfield MM, Jacobsen SJ, Burnett JC Jr, et al. Burden of systolic and diastolic ventricular dysfunction in the community: appreciating the scope of the heart failure epidemic. JAMA 2003;289:194-202.

6. Hurrell DG, Nishimura RA, Ilstrup DM, et al. Utility of preload alteration in assessment of left ventricular filling pressure by Doppler echocardiography: a simultaneous catheterization and Doppler echocardiographic study. J Am Coll Cardiol 1997:30:459-67.

7. Appleton CP, Galloway JM, Gonzalez MS, et al. Estimation of left ventricular filling pressures using two-dimensional and Doppler echocardiography in adult patients with cardiac disease. Additional value of analyzing left atrial size, left atrial ejection fraction and the difference in duration of pulmonary venous and mitral flow velocity at atrial contraction. J Am Coll Cardiol 1993;22:1972-82.

8. Simek CL, Feldman MD, Haber HL, et al. Relationship between left ventricular wall thickness and left atrial size: comparison with other measures of diastolic function. J Am Soc Echocardiogr 1995;8:37-47.

9. Lester SJ, Ryan EW, Schiller NB, et al. Best method in clinical practice and in research studies to determine left atrial size. Am J Cardiol 1999;84:829-32.

10. Tsang TS, Barnes ME, Gersh BJ, et al. Left atrial volume as a morphophysiologic expression of left ventricular diastolic dysfunction and relation to cardiovascular risk burden. Am J Cardiol 2002;90:1284-9.

11. Sabharwal N, Cemin R, Rajan K, et al. Usefulness of left atrial volume as a predictor of mortality in patients with ischemic cardiomyopathy. Am J Cardiol 2004;94:760-3.

12. Beinart R, Boyko V. Schwammenthal $E$, et al. Long-term prognostic significance of left atrial volume in acute myocardial infarction. J Am Coll Cardiol 2004;44:327-34.

13. Lim TK, Ashrafian H, Dwivedi G, et al. Increased left atrial volume index is an independent predictor of raised serum natriuretic peptide in patients with suspected heart failure but normal left ventricular ejection fraction: implication for diagnosis of diastolic heart failure. Eur J Heart Fail 2006;8:38-45.

14. Galasko GI, Lahiri A, Senior R. Portable echocardiography: an innovative tool in screening for cardiac abnormalities in the community. Eur J Echocardiogr 2003:4:119-27.

15. Foster $\mathbf{E}$, Cahalan MK. The search for intelligent quantitation in echocardiography: "eyeball," "trackball" and beyond. J Am Coll Cardiol 1993;22:848-50.

16. Pritchett AM, Jacobsen SJ, Mahoney DW, et al. Left atrial volume as an index of left atrial size: a population-based study. J Am Coll Cardiol 2003;41:1036-43.

17. Masani M, Wharton G, Allen J, et al. British Society of Echocardiography: guidelines for chamber quantification. 2008. Available at http://www.bsecho.org/ Guidelines\%20for\%20Chamber\%200uantification.pdf (accessed 3 May 2009).

18. Cohn JN, Johnson GR, Shabetai R, et al. Ejection fraction, peak exercise oxygen consumption, cardiothoracic ratio, ventricular arrhythmias, and plasma norepinephrine as determinants of prognosis in heart failure. The V-HeFT VA Cooperative Studies Group. Circulation 1993;87(Suppl):VI5-16.

19. Solomon SD, Anavekar N, Skali H, et al. Influence of ejection fraction on cardiovascular outcomes in a broad spectrum of heart failure patients. Circulation 2005; 112:3738-44.

20. White HD, Norris RM, Brown MA, et al. Left ventricular end-systolic volume as the major determinant of survival after recovery from myocardial infarction. Circulation 1987:76:44-51. 
21. Tsang TS, Abhayaratna WP, Barnes ME, et al. Prediction of cardiovascular outcomes with left atrial size: is volume superior to area or diameter? J Am Coll Cardiol 2006;47:1018-23.

22. Yamamoto $\mathbf{K}$, Nishimura RA, Chaliki HP, et al. Determination of left ventricular filling pressure by Doppler echocardiography in patients with coronary artery disease: critical role of left ventricular systolic function. J Am Coll Cardiol 1997:30:1819-26.

23. Pritchett AM, Mahoney DW, Jacobsen SJ, et al. Diastolic dysfunction and left atrial volume: a population-based study. J Am Coll Cardiol 2005:45:87-92.

24. Benjamin EJ, D'Agostino RB, Belanger AJ, et al. Left atrial size and the risk of stroke and death. The Framingham Heart Study. Circulation 1995;92:835-41.

25. Rossi A, Cicoira M, Bonapace S, et al. Left atrial volume provides independent and incremental information compared with exercise tolerance parameters in patients with heart failure and left ventricular systolic dysfunction. Heart 2007:93:1420-5
26. Whalley GA, Wright SP, Pearl A, et al. Prognostic role of echocardiography and brain natriuretic peptide in symptomatic breathless patients in the community. Eur Heart $J$ 2008;29:509-16.

27. Lang RM, Bierig M, Devereux RB, et al. Recommendations for chamber quantification: a report from the American Society of Echocardiography's Guidelines and Standards Committee and the Chamber Quantification Writing Group, developed in conjunction with the European Association of Echocardiography, a branch of the European Society of Cardiology. J Am Soc Echocardiogr 2005;18:1440-63.

28. Lim TK, Hayat SA, Gaze D, et al. Independent value of echocardiography and Nterminal pro-natriuretic peptide for the prediction of major outcomes in patients with suspected heart failure. Am J Cardiol 2007;100:870-5.

29. Vourvouri EC, Poldermans D, Deckers JW, et al. Evaluation of a hand carried cardiac ultrasound device in an outpatient cardiology clinic. Heart 2005;91:171-6.

30. Vourvouri EC, Schinkel AF, Roelandt JR, et al. Screening for left ventricular dysfunction using a hand-carried cardiac ultrasound device. Eur J Heart Fail 2003:5:767-74.

\section{Images in cardiology}

\section{Rare case of blunt chest trauma induced left main and LAD dissection in association with anomalous RCA origin}

Myocardial infarction is a rare complication of blunt chest trauma. A 47-year-old man who was a chronic smoker presented in the emergency department with multiple injuries of the face, arms, right leg and chest after a motor vehicle accident. The patient's electrocardiogram showed ST elevation in precordial leads V1-V6. On $x$-ray examination of the chest, signs of pulmonary venous congestion were seen. Echocardiography showed extensive wall motion abnormality in the region of the left anterior descending artery (LAD) with a left ventricular ejection fraction of $25 \%$. Thrombolytic treatment was not given in view of the multiple injuries. The patient's coronary angiogram showed dissection of the left main artery and the LAD with total occlusion of the proximal LAD (panel). The right coronary artery (RCA) was anomalously originating from the left coronary sinus and was normal. LAD dissection in relation to blunt chest trauma has been reported but extensive dissection of the left main artery extending into the LAD is extremely rare. ${ }^{1}$

\section{G Goyal, G Singh, R Kapoor}

gajindergoyal@yahoo.co.in

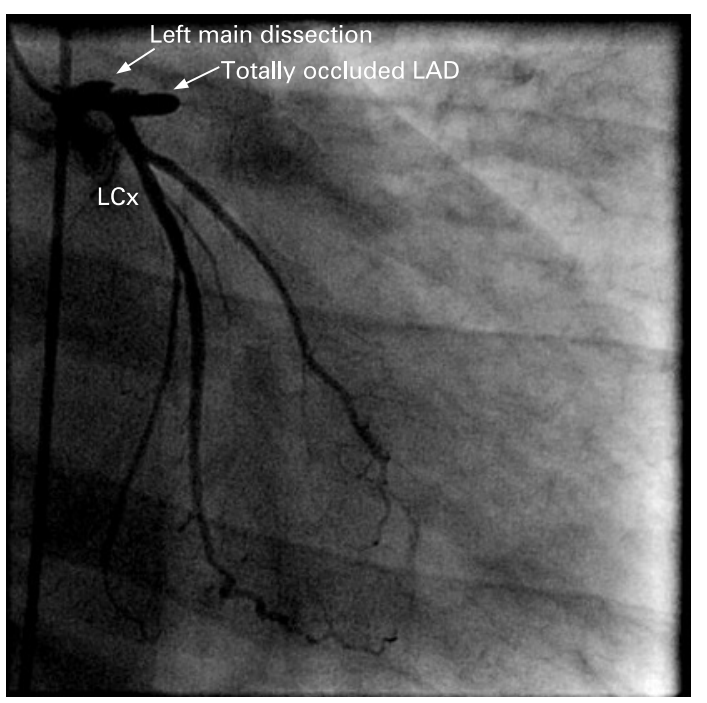

- Additional videos are published online only at http://heart.bmj.com/content/vol95/ issue 14

Heart 2009;95:1178. doi:10.1136/hrt.2009.168914

\section{REFERENCE}

1. Goktekin 0, Unalir A, Gorenek B, et al. Traumatic total occlusion of left main coronary artery caused by blunt chest trauma. J Invasive Cardiol 2002;14:463-5. 